\title{
Recent results from ATLAS: Onia, heavy-flavor, and more
}

\author{
Martin Spousta \\ on behalf of the ATLAS Collaboration \\ Faculty of Mathematics and Physics, Charles University \\ V Holešovičkách 2, Prague 180 00, Czech Republic \\ martin.spousta@mff.cuni.cz
}

\begin{abstract}
In this short report we review the recent results obtained using the ATLAS experiment at the LHC on quarkonia and heavy flavor measurements in heavy-ion collisions.
\end{abstract}

Keywords: Quarkonia, Heavy-flavor, Heavy-ion collisions, ATLAS, LHC

In this short report we review the recent results obtained using the ATLAS experiment [1] at the LHC on quarkonia and heavy flavor measurements in heavy-ion collisions. The text is divided into two sections. The first section briefly discusses the final state effects in the production of quarkonia and heavy flavor and it describes the measurements of suppression and flow of quarkonia and heavy-flavor in $\mathrm{Pb}+\mathrm{Pb}$ collisions. The second section discusses the initial state effects and describes the measurements done in $p+\mathrm{Pb}$ collisions.

\section{Suppression and flow of quarkonia and heavy-flavor in $\mathrm{Pb}+\mathrm{Pb}$ collisions}

It was predicted a long time ago that the Debye screening of the quark colour charge in a hot quark-gluon plasma (QGP) would lead to a dissociation of quarkonium bound state in the medium, when the Debye length becomes smaller than the quarkonium binding radius [2]. While the strong suppression of quarkonia in nucleus-nucleus collisions is firmly established experimental fact, its interpretation remains open. Besides initial state effects and the interaction with co-moving hadrons, which are discussed in the context of $p+\mathrm{Pb}$ measurements in Section 2, it is also the parton energy loss which was recently suggested as an alternative physics mechanism for the observed quarkonia suppression [3, 4]. To help address the origin of the quarkonium suppression, ATLAS published a measurement [5] of prompt charmonia, originating from the formation of $c \bar{c}$ bound state which follows immediately after the hard process, and non-prompt charmonia, originating from $b$-hadron decays. In that measurement, prompt and non-prompt per-event yields, non-prompt fraction and nuclear modification factors, $R_{\mathrm{AA}}$, of the $J / \psi$ and $\psi(2 S)$ is performed. The measurement is performed 

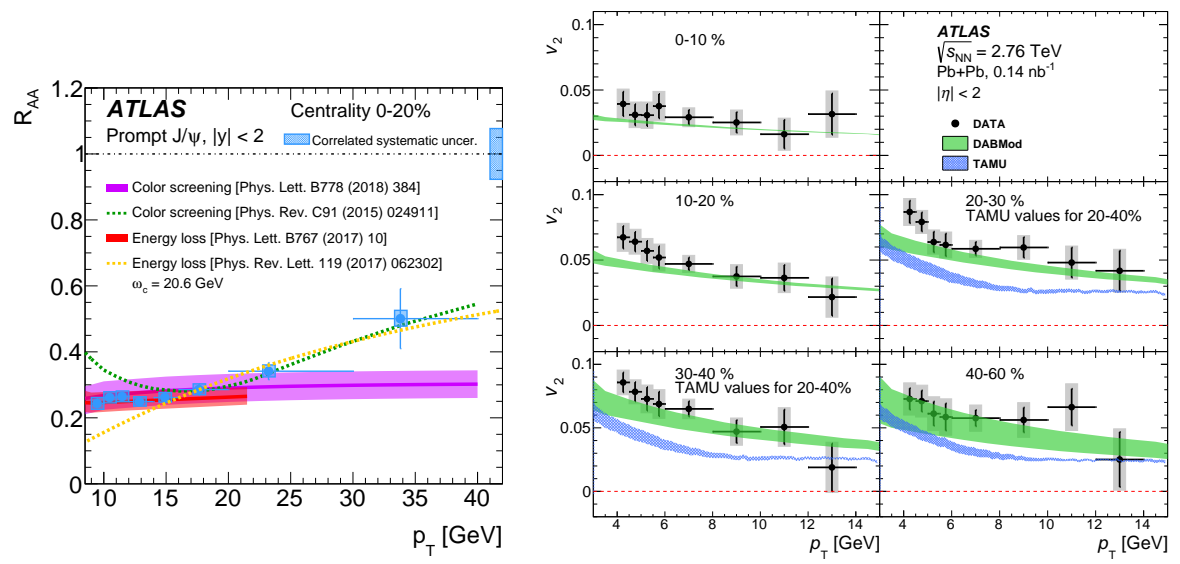

Fig. 1. Left: Comparison of $R_{\mathrm{AA}}$ for prompt $J / \psi$ production with different theoretical models. The statistical uncertainty of each point is indicated by a narrow error bar. The error box plotted with each point represents the uncorrelated systematic uncertainty, while the shaded error box at $R_{\mathrm{AA}}=1$ represents correlated scale uncertainties. Plot taken from Ref. [5]. Right: Comparison of the $\mathrm{Pb}+\mathrm{Pb}$ heavy-flavor muon v2 with calculations from the TAMU [6] and DABMod [7] models. Each panel represents a different centrality interval. For the $20-30 \%$ and $30-40 \%$ centrality intervals, the plotted TAMU values correspond to the $20-40 \%$ centrality interval. For the data, the error bars and shaded bands represent statistical and total uncertainties, respectively. For the model calculations, the bands represent theoretical systematic uncertainties. Plot taken from Ref. [8].

in the dimuon decay channel in $\mathrm{Pb}+\mathrm{Pb}$ collisions at $\sqrt{s_{N N}}=5.02 \mathrm{TeV}$, for quarkonia with transverse momentum $9<p_{\mathrm{T}}<40 \mathrm{GeV}$ and rapidity $|y|<2.0$.

Strong suppression of prompt and non-prompt $J / \psi$ and $\psi(2 S)$ mesons is observed in $\mathrm{Pb}+\mathrm{Pb}$ data. In the $0-10 \%$, the $R_{\mathrm{AA}}$ of prompt $J / \psi$ is approximately 0.2 for $p_{\mathrm{T}}=10 \mathrm{GeV}$ and it grows and achieves a value of 0.3 at $p_{\mathrm{T}} \approx 25 \mathrm{GeV}$. The $R_{\mathrm{AA}}$ of non-prompt $J / \psi$ in $0-10 \%$ is approximately 0.3 and remains independent of $p_{\mathrm{T}}$ within the full $p_{\mathrm{T}}$ range of this measurement. The dependence of the $R_{\mathrm{AA}}$ on centrality is approximately the same for prompt and non-prompt $J / \psi$. The ratio of $\psi(2 S)$ to $J / \psi$ meson production is measured for both the prompt and non-prompt mesons, and as a function of centrality. Values consistent with unity are measured for the non-prompt mesons, while the values observed for the prompt mesons are below unity. The left panel of Figure 1 shows a comparison of the $J / \psi R_{\mathrm{AA}}$ measured in $0-20 \%$ central collisions with theoretical calculations.

ATLAS also measured the elliptic flow coefficient $\left(v_{2}\right)$ of prompt and nonprompt $J / \psi$ in $\mathrm{Pb}+\mathrm{Pb}$ collisions [9]. The measurement is done using similar techniques and kinematic cuts and using the same dataset as used for the measurement of the $R_{\mathrm{AA}}$ of $J / \psi$ and $\psi(2 S)$. In that measurement, the $v_{2}$ coefficient is evaluated relative to the event plane and the results are presented as a func- 
tion of $p_{\mathrm{T}}, y$ and centrality. It is found that both prompt and non-prompt $J / \psi$ mesons have non-zero elliptic flow. Prompt $J / \psi v_{2}$ is found to decrease as a function of $p_{\mathrm{T}}$, while the non-prompt $J / \psi$ is found to be, with limited statistical significance, consistent with a flat behaviour over the studied kinematic region. There is no observed dependence on $y$ or centrality.

The suppression and the azimuthal anisotropy was measured also for muons from heavy-flavor decays in $2.76 \mathrm{TeV} \mathrm{Pb}+\mathrm{Pb}$ collisions [8]. The measurement is performed over the muon transverse momentum range $4<p_{\mathrm{T}}<14 \mathrm{GeV}$. Backgrounds arising from in-flight pion and kaon decays, hadronic showers, and misreconstructed muons is statistically removed using a template-fitting procedure. The resulting $R_{\mathrm{AA}}$ is observed to be independent of $p_{\mathrm{T}}$ within uncertainties. For the $10 \%$ most central $\mathrm{Pb}+\mathrm{Pb}$ events, the measured $R_{\mathrm{AA}}$ is approximately 0.35 indicating a clear suppression of production of heavy-flavor muons in $\mathrm{Pb}+\mathrm{Pb}$ collisions.

The azimuthal modulation of the heavy-flavor muon yields is measured and the associated Fourier coefficients $v_{n}$ for $n=2,3$ and 4 are given as a function of $p_{\mathrm{T}}$ and centrality. Significant $v_{2}$ values up to about 0.08 are observed at $p_{\mathrm{T}}=$ $4 \mathrm{GeV}$. In the $10-20 \%, 20-30 \%$, and $30-40 \%$ intervals, the $v_{2}$ decreases with $p_{\mathrm{T}}$ but is still significant at $10 \mathrm{GeV}$. At fixed $p_{\mathrm{T}}$, the $v_{2}$ values show a systematic variation with centrality which is typical of elliptic-flow measurements. For most centrality intervals, $v_{3}$ also decreases with increasing $p_{\mathrm{T}}$ over the $4-8 \mathrm{GeV} p_{\mathrm{T}}$ range. At a given $p_{\mathrm{T}}$ and centrality, the $v_{3}$ values are smaller than the $v_{2}$ values by a factor of $2-4$. The $v_{3}$ values show a much weaker centrality dependence than the $v_{2}$. The right panel of Figure 1 shows a comparison of heavy-flavor muon $v_{2}$ in five centrality bins with theory calculations.

\section{Quarkonia and heavy flavor in $p+P b$ collisions}

In order to understand quarkonium suppression in $\mathrm{Pb}+\mathrm{Pb}$ collisions it is necessary to disentangle effects due to interaction between quarkonium and the QGP medium from those that can be ascribed to initial state effects, such as: modifications of the nuclear parton distribution functions, parton saturation effects in the incident nucleus, and parton energy loss through interactions with the nuclear medium of the initial state. These effects can be accessed in $p+\mathrm{Pb}$ collisions where a large region of hot QGP is a priori not expected to occur. At the same time, modification of quarkonia production is also expected even in $p+\mathrm{Pb}$ collisions to originate from final state effects, namely from the absorption of the heavy quark-antiquark pair through interactions with the co-moving hadrons. For a review of these physics mechanisms see e.g. Ref. [10].

To help disentangle the role of the above mentioned phenomena, ATLAS measured the production cross sections of five quarkonium states, $J / \psi, \psi(2 S)$, and $\Upsilon(n S)(n=1,2,3)$ in $p+\mathrm{Pb}$ collisions at $5.02 \mathrm{TeV}$ with an integrated luminosity of $28 \mathrm{nb}^{-1}$ [11]. The resulting nuclear modification factor, $R_{p \mathrm{~Pb}}$, is found to be consistent with unity for prompt and non-prompt $J / \psi$ for the kinematic range of the measurement, $8<p_{\mathrm{T}}<40 \mathrm{GeV}$. The prompt $\psi(2 S)$ production 

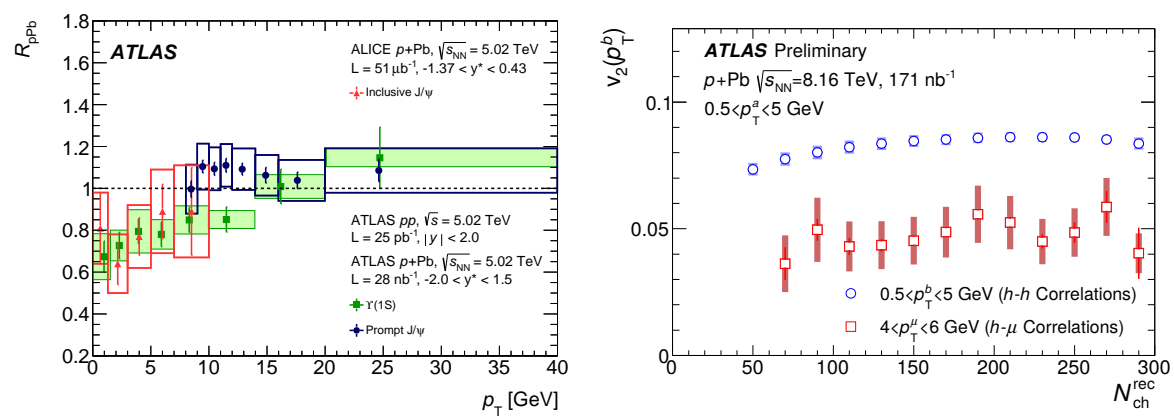

Fig. 2. Left: Nuclear modification factor, $R_{p \mathrm{~Pb}}$, as a function of $p_{\mathrm{T}}$ for $\Upsilon(1 S)$ (in green) and prompt $J / \psi$ (in blue). The vertical error bars cover the statistical uncertainties and horizontal error bars represent the bin size. The horizontal position of data point indicates the mean of the weighted $p_{\mathrm{T}}$ distribution. The vertical size of colored boxes underneath the data points represent the systematic uncertainties. The $R_{p \mathrm{~Pb}}$ of inclusive $J / \psi$ measured by ALICE from [12] is also shown in red. Figure taken from Ref. [11]. Right: The $v_{2}$ as a function of number of reconstructed charged particles $\left(N_{\mathrm{ch}}^{\mathrm{rec}}\right)$ obtained from the template fits to hadron-hadron (circles) and to hadron- $\mu$ correlations (squares). The error bars and shaded bands indicate statistical and systematic uncertainties, respectively. For the hadron-hadron correlations, the statistical errors are too small to be seen. Figure taken from Ref. [13].

is suppressed with respect to prompt $J / \psi$ production in $p+\mathrm{Pb}$ collisions with a significance of one standard deviation.

The $R_{p \mathrm{~Pb}}$ for $\Upsilon(1 S)$ is measured for $p_{\mathrm{T}}<40 \mathrm{GeV}$ and is found to be smaller than unity at $p_{\mathrm{T}}<15 \mathrm{GeV}$, increasing with $p_{\mathrm{T}}$ and becoming compatible with unity at high $p_{\mathrm{T}}$. The production of $\Upsilon(2 S)$ and $\Upsilon(3 S)$ is found to be suppressed with respect to $\Upsilon(1 S)$ in the integrated kinematic ranges of $p_{\mathrm{T}}<40 \mathrm{GeV}$ and $-2<y^{*}<1.5$ in $p+\mathrm{Pb}$ collisions with significance at the level of two standard deviations. Both the prompt $\psi(2 S)$ to $J / \psi$ and $\Upsilon(2 S)$ to $\Upsilon(1 S)$ double ratios show decreasing trend with increasing centrality of $p+\mathrm{Pb}$ collisions. The left panel of Figure 2 shows a compilation of results on $R_{p \mathrm{~Pb}}$ measured as a function of $p_{\mathrm{T}}$ for $\Upsilon(1 S)$ and prompt $J / \psi$.

The initial stage effects can also be studied using measurements of heavy quarks. ATLAS measured prompt $D^{0}$ mesons $\left(D^{0}\right.$ and $\left.\bar{D}^{0}\right)$ and $D^{*}$ mesons (which are not coming from the sequential decays of $b$-hadrons) using $p+\mathrm{Pb}$ collisions at $8.16 \mathrm{TeV}$ with an integrated luminosity of $76.3 \mu \mathrm{b}^{-1}$ [14]. The $D^{0}$ and $D^{*}$ mesons are reconstructed in the decay channels with kaons in the range of transverse momentum $3<p_{\mathrm{T}}<30 \mathrm{GeV}$ and $5<p_{\mathrm{T}}<30 \mathrm{GeV}$, respectively. The relative $D$ meson productions at forward and backward center of mass rapidities is studied via the forward $(0<y<0.5)$ to backward $(-0.5<y<0)$ production ratio, $R_{F B}$, as a function of transverse momentum. No significant forward-backward asymmetry for prompt $D^{0}$ and $D^{*}$ mesons is observed. The azimuthal correlations between inclusive $D^{*}$ mesons and charged particles is also 
measured. The second-order harmonic coefficients for $D^{*}$-hadron correlations are extracted using template fits to remove the "non-flow" contributions. The $D^{*}$-hadron correlations are broadly consistent with the measurement of muonhadron correlations.

A detailed measurement of long-range pseudorapidity correlations between muons and charged particles was done using $p+\mathrm{Pb}$ data at $8.16 \mathrm{TeV}$ [13]. The template fitting method is used to obtain the second-order Fourier coefficient, $v_{2,2}$, corresponding to the genuine long-range correlations. The $v_{2,2}$ is factorized to obtain the single-particle anisotropy coefficient, $v_{2}$, of the muons. The right panel of Figure 2 shows a comparison of hadron- $\mu$ and hadron-hadron $v_{2}$. Significant $v_{2}$ values are observed for muons over the transverse momentum interval $4<p_{\mathrm{T}}<8 \mathrm{GeV}$ and over the range of the charged particle multiplicity of $100-300$. The muon- $v_{2}$ values are largest at $4 \mathrm{GeV}$ and decrease with increasing $p_{\mathrm{T}}$. Given that nearly all the prompt muons over this $p_{\mathrm{T}}$ range are produced from decays of heavy-flavor particles, these results confirm a significant azimuthal anisotropy in the distribution of heavy-flavor particles produced in $p+\mathrm{Pb}$ collisions.

\section{Conclusions}

The ATLAS Experiment at the LHC provides a wealth of new results on quarkonia and heavy flavor measurements in $\mathrm{Pb}+\mathrm{Pb}$ and $p+\mathrm{Pb}$ collisions. The new results from $\mathrm{Pb}+\mathrm{Pb}$ collisions provide a precise quantification of strong suppression effects and flow phenomena. The new results from $p+\mathrm{Pb}$ collisions should allow constraints to be put on the origin of flow phenomena in small collision systems and quantify the role of initial state effects in the studied data.

\section{Acknowledgment}

This work was supported by Grant Agency of the Czech Republic under grant 1812859Y, by the Ministry of Education, Youth and Sports of the Czech Republic under grant LTT 17018, and by Charles University grant UNCE/SCI/013.

Copyright 2019 CERN for the benefit of the ATLAS Collaboration. CC-BYNC-ND-4.0 license.

\section{References}

1. ATLAS Collaboration. The ATLAS Experiment at the CERN Large Hadron Collider. JINST, 3:S08003, 2008.

2. T. Matsui and H. Satz. $J / \psi$ Suppression by Quark-Gluon Plasma Formation. Phys. Lett., B178:416-422, 1986.

3. Franois Arleo. Quenching of Hadron Spectra in Heavy Ion Collisions at the LHC. Phys. Rev. Lett., 119(6):062302, 2017. 
4. Martin Spousta. On similarity of jet quenching and charmonia suppression. Phys. Lett., B767:10-15, 2017.

5. ATLAS Collaboration. Prompt and non-prompt $J / \psi$ and $\psi(2 \mathrm{~S})$ suppression at high transverse momentum in $5.02 \mathrm{TeV} \mathrm{Pb}+\mathrm{Pb}$ collisions with the ATLAS experiment. Eur. Phys. J., C78(9):762, 2018.

6. Min He, Rainer J. Fries, and Ralf Rapp. Heavy Flavor at the Large Hadron Collider in a Strong Coupling Approach. Phys. Lett., B735:445-450, 2014.

7. Caio A. G. Prado, Jacquelyn Noronha-Hostler, Roland Katz, Alexandre A. P. Suaide, Jorge Noronha, Marcelo G. Munhoz, and Mauro R. Cosentino. Event-byevent correlations between soft hadrons and $D^{0}$ mesons in $5.02 \mathrm{TeV} \mathrm{PbPb}$ collisions at the CERN Large Hadron Collider. Phys. Rev., C96(6):064903, 2017.

8. ATLAS Collaboration. Measurement of the suppression and azimuthal anisotropy of muons from heavy-flavor decays in $\mathrm{Pb}+\mathrm{Pb}$ collisions at $\sqrt{s_{\mathrm{NN}}}=2.76 \mathrm{TeV}$ with the ATLAS detector. Phys. Rev., C98(4):044905, 2018.

9. ATLAS Collaboration. Prompt and non-prompt $J / \psi$ elliptic flow in $\mathrm{Pb}+\mathrm{Pb}$ collisions at $\sqrt{s_{N N}}=5.02$ Tev with the ATLAS detector. Eur. Phys. J., C78(9):784, 2018 .

10. A. Andronic et al. Heavy-flavour and quarkonium production in the LHC era: from protonproton to heavy-ion collisions. Eur. Phys. J., C76(3):107, 2016.

11. ATLAS Collaboration. Measurement of quarkonium production in protonlead and protonproton collisions at $5.02 \mathrm{TeV}$ with the ATLAS detector. Eur. Phys. J., C78(3):171, 2018.

12. ALICE Collaboration. Rapidity and transverse-momentum dependence of the inclusive $\mathrm{J} / \psi$ nuclear modification factor in $\mathrm{p}-\mathrm{Pb}$ collisions at $\sqrt{s_{N N}}=5.02 \mathrm{TeV}$. JHEP, 06:055, 2015.

13. ATLAS Collaboration. Measurement of the long-range pseudorapidity correlations between muons and charged-particles in $\sqrt{s_{\mathrm{NN}}}=8.16 \mathrm{TeV}$ proton-lead collisions with the ATLAS detector. ATLAS-CONF-2017-006.

14. ATLAS Collaboration. $D$ meson production and long-range azimuthal correlation in $8.16 \mathrm{TeV} p+\mathrm{Pb}$ collisions with ATLAS. ATLAS-CONF-2017-073. 